\title{
Análisis de un Brote de Diarrea por E. Coli 0142 en Recién Nacidos
}

\author{
Dr. Enrique Hering. A. ${ }^{1}$, Q.F. Eliana Marambio L. ${ }^{2}$, \\ Dra. Valeria Prado J.3 \\ Enteropathogenic Type 0142, E. Coli \\ in Diarrhea Among Neonates
}

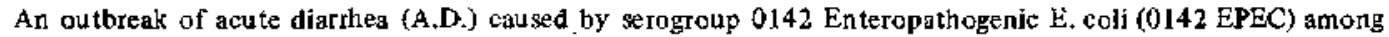
neonates from the wards of a general hospital is described on clinical and epidemiological grounds. Seventy patients were affected from January through Septembex 1982, all of them had negative stool cultures for Salmonella and Shigella sepecies. Fourteen of twenty two neonates specifically investigated for E. coli rendered positive isolations, in all cases 0142 EPEC. In this otubreak $92,8 \%$ of patients were preterm and $85,7 \%$ of the whole group suffered severe symptoms, with dehydration and metabolic acidosis. Mortality rate was $2,9 \%$ The relationships between EPEC infection and the relatively inmunodeficient host represented by the newborn are discussed, Breat feeding reduced the incidence and severity of diarthea and the complications of infection.
\end{abstract}

La aparición esporádica de brotes epidémicos de diarrea aguda en unidades de recién nacidos es un hecho conocido y cuando ello ocurre puede adquirir caracteres dramáticos, ya que generalmente afecta al grupo de más alto riesgo que son los recién nacidos de bajo peso.

En la unidad de recién nacidos del Hospital del Salvador se han detectado en el pasado brotes por Salmonella bredeney ${ }^{1}$ y por una cepa enterotoxigénica de Bacillus cereus ${ }^{2}$.

1 Jefe Unidad Neonatología Hospital El Salvador.

2 Institu to Salud Pública (I.S.P.).

3 Jefe Unidad Mierobiologia, Div. Ciencias Médicas Oriente, Fac. Medicina. Universidad de Chile.
Desde Enero de 1982, se observó en la unidad, un aumento de las infecciones intrahospitalarias con predominio de cuadros de diarrea aguda, que persistieron en el transcurso de los meses, sin poder definir la identidad exacta del agente etiológico. En Julio 1982, el número de casos aumentó en forma significativa, por lo cual se pidió la colaboración del Instituto de Salud Pública (I.S.P.) lográndose aislar una cepa de E. coli enteropatógeno clásico (ECEP) serogrupo 0142.

Existen numerosas comunicaciones respecto a diferentes. serogrupos de ECEP involucrados en brotes en centros cerrados ${ }^{3 \cdot 4}$. En la diarrea endémica de lactantes en Chile los serogrupos más frecuentes son: $0111-055-0119-086$. 
$0125-0128^{5 \cdot 6 \cdot 7-8-9}$; sin embargo no hay referencias a episodios epidémicos en recién nacidos por el serogrupo 0142, por lo que no siempre se incluye el correspondiente antisuero entre los utilizados en el laboratorio de los hospitales. Por ello hemos considerado de interés analizar las caracteristicas clinicas y epidemiológicas de este brote.

\section{MATERIAL Y METODO}

Se analizaron las características clínicas de 70 recién nacidos (R.N.) que sufrieron diarrea aguda (D.A.) mientras estaban hospitalizados en la Unidad de Neonatología del Hospital del Salve. dor en el periodo comprendido entre Enero a Septiembre 1982. Para los fines de este estudio se consideró como diarrea aguda la presencia de 30 măs deposiciones al dia de consistencia alterada (liquidas, disgregadas, mucosas) acompañada o no de deshidratación.

Con el objeto de determinar la etiología de la enfermedad se realizaron coprocultivos seriados en todos los casos. orientados al aislamiento de Salmonella y Shigella utilizando las técnicas recomendadas por Edwards y Ewing ${ }^{10}$. Desde Julio de 1982 se agregó tipificación de serogrupos clásicos de ECEP y cultivo de Bacillus cereus y Staphilococus aureus en deposiciones en 22 pacientes, siguiendo técnicas normalizadas ${ }^{1}$.

Además se hicieron estudios bacteriológicos de las mamaderas del servicio de leche de la unidad, tanto de leches comercjales ( 10 mamaderas) como maternas (2 mamaderas), recolectadas al azar en un plazo de 3 semanas, realizando recuento total de aerobios mesófilos, de Bacillus cereus $y$ de coliformes totales y fecales según normas técnicas internacionales ${ }^{12}$.

Se realizaron estudios para detectar rotavirus en 10 pacientes, (en el mes de Julio) utilizando el metodo de electroforesis en gel de agarosa. acrilamida $^{13}$.

\section{RESULTADOS}

La Tabla 1 detalla la tasa de ataque de diarrea aguda (D.A.) en los pacientes hospitalizados en lat unidad de neonatología a lo largo de los meses de Enero a Octubre. Observamos que desde una tasa mantenida, de 1,5 a $3,5 \%$ se produjo una eleva. ción a $8.9 \%$ en el mes de 3 ulio, la que después de identificar el problema y tomar las medidas de control correspondientés, bajó a cero en Septiembre y Octubre (Fig. 1 ).

Tabla 1.

Tasa de atayue de diarrea aguda

$\begin{array}{cccc}\text { Mes } & \begin{array}{c}\text { No pacientes } \\ \text { hospitalizados }\end{array} & \begin{array}{c}\text { Casos nuevos } \\ \text { de diartea } \\ \text { No }\end{array} & \% \\ \text { I } & 261 & 7 & 2,68 \\ \text { II } & 269 & 5 & 1,8,5 \\ \text { III } & 230 & 8 & 3,5 \\ \text { IV } & 277 & 4 & 1,44 \\ \text { V } & 273 & 4 & 1,5 \\ \text { VI } & 321 & 5 & 1,8 \\ \text { VII } & 271 & 24 & 8,9 \\ \text { VIII } & 259 & 13 & 5,02 \\ \text { IX } & 434 & 0 & 0 \\ \text { X } & 320 & 0 & 0\end{array}$

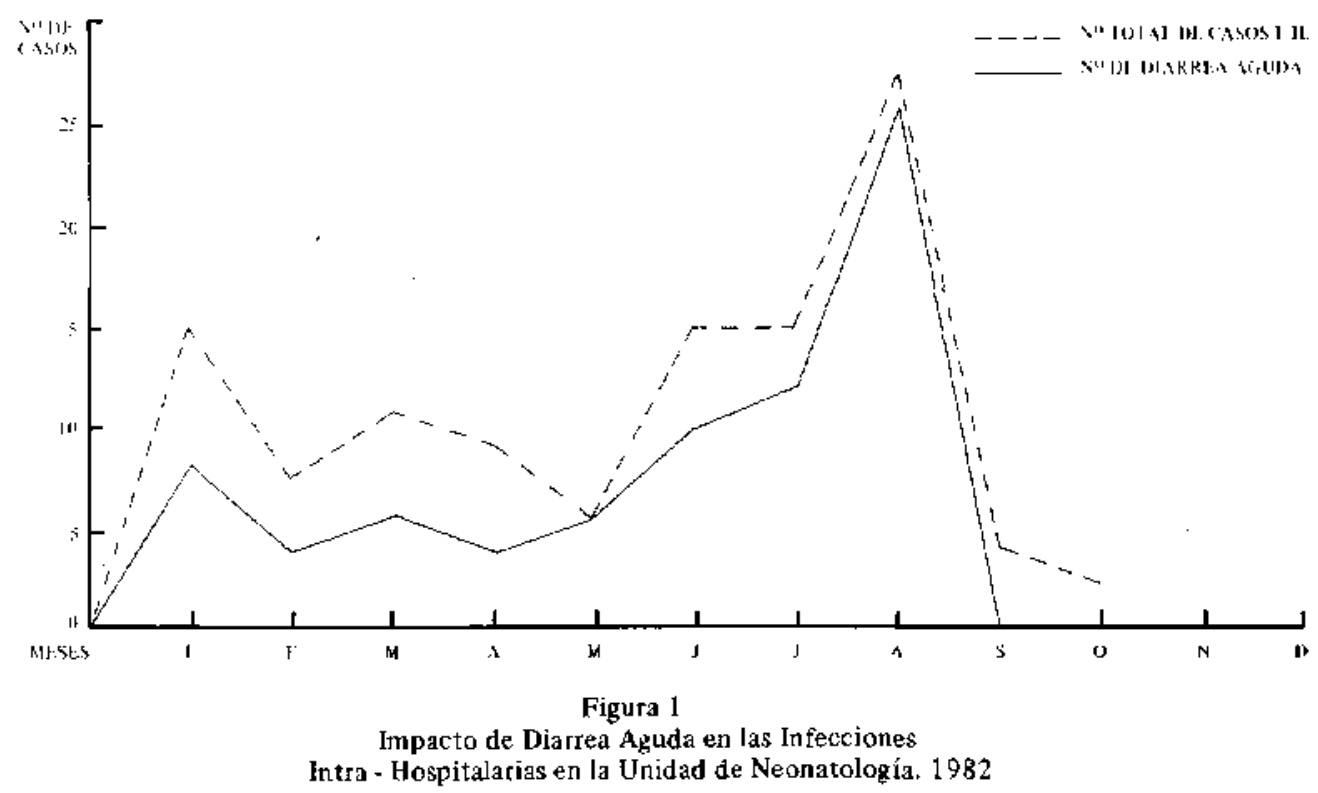

Incidencia de infecciones intra hospitalarias en la Unidad de Neonatología entre los meses de Enero a Octubre (línea punteada) en línca continua el número de casos de diarrea aguda. La diarrea constituyó el problema intrahospitalario más importante haciéndose crítico en el mes de Julio. 
La Tabla 2 señala la distribución de los casos de D.A. en las diferentes secciones de la unidad de recién nacidos; llamó la atención que la mayor concentración de casos nuevos se produjo en las secciones denominadas Mínimo I, II e Intermedio, a las que ingresaron los recién nacidos de más bajo peso. Los casos de diarrea aguda observados en la Unidad de Tratamiento Intensivo (UTJ) correspondieron a pacientes trasladados desde las mencionadas secciones.

Tabla 2.

Distribución de los

casos de D.A. en diferentes Secciones

$\begin{array}{lcr}\text { Sala } & \text { No casos } & \% \\ \text { Mínimo I } & 25 & 35,7 \\ \text { Mínimo II } & 5 & 7,2 \\ \text { Intermedio I } & 16 & 22,8 \\ \text { Internedio II } & 14 & 20,0 \\ \text { U.T.I. } & 10 & 14,3 \\ & & \\ & 70 & 100\end{array}$

La mayor incidencia de D.A. en el presente brote se observó en los recién nacidos de pretérmino (R.N.Pr.); del total de casos (70), un 92,8\% eran R.N.Pr. y sólo el 7,2\% de término.

De estos pacientes la mayoría, $51 \quad(72,8 \%)$, presentaron síntomas graves con deshidratación moderada o intensa acidosis metabólica persistente; 4 pacientes $(5,7 \%)$ evolucionaron hacia una enterocolitis necrotizante (E.C.N.); en 5 R.N. $(7,2 \%)$ se encontró septicemia además de diarrea, aislándose en los hemocultivos el mismo $\mathrm{E}$. coli 0142 en 1 caso, Klebsiella pneumoniae en 3 y Pseudomonas aeruginosa en un paciente. En 10 R.N. con infección por E. coli 0142 la diarrea cursó sin deshidratación y con pocas repercusiones en el estado general. De los 70 R.N. con diarrea aguda, la evolución fue satisfactoria en 64 $(91,4 \%) ; 4$ de ellos $(5,7 \%)$ necesitaron tratamiento quirúrgico por E.C.N. y 2 pacientes fallecieron $(2,9 \%)$, los seis pertenecían al grupo en que se comprobó septicemia además de la infección entérica.

La investigación de Salmonella y Shigella en los 70 R.N. con diarrea aguda resultó negativa. De 22 pacientes en que se tipificaron las EC se aisló el tipo 0142 en 14, siendo negativos los cultivos para B. cereus y $\mathrm{S}$. aureus.

En los 10 nifios con D.A. seleccionados al azar para buscar rotavirus en las deposiciones, los resultados fueron negativos.

El estudio de sensibilidad in vitro de las cepas de E. coli 0142 aisladas mostró en todas un patrón común: Sensibilidad a Furazolidona, Sulfato de colistin, Amikacina.y Tetraciclina. Resis- tencia a Neomicina, Cloranfenicol, Ampicilina y Cotrimoxazol. Los cultivos de mamaderas con ambos tipos de leche fueron negativos para $B$. cereus, $\mathbf{S}$. aureus y coliformes totales y fecales. EI recuento total de aerobios mesófilos dio cifras desde $<1 \times 10^{3}$ hasta $3 \times 10^{5}$ colonias $/ \mathrm{ml}$.

La Tabla 3 muestra las relaciones entre el tipo de alimentación y las de complicaciones en los 70 niños afectados por este brote. Las últimas ocurrieron con mayor frecuencia en pacientes que recibian alimentación artificial exclusiva. La diferencia tiene significación estadistica.

Tabla 3.

Correlación entre incidencia de diarrea aguda y tipo de alimentación

Síntomas

Diar̀tea aguda

Diarrea + Septicemia

Diarrea con E.C.N.*

TOTAL

47

Alimentación
Leche
Comercial
Matema
(L.C.)
(L.M.)

(LA.)

*E.C. $\mathrm{N} .=$ enterocolitis necrotizan te
$\mathrm{X}^{2}$ calc $=29,66$

\section{COMENTARIO}

Las evidencias sobre la capacidad que tienen determinados E. coli de producir diarrea en niños pequeños, ya sea en forma endémica o en brotes epidémicos son bastante antiguas y datan de la década del 40 con algunos trabajos ingleses ${ }^{13-3}$. Posteriormente Kauffmann ideó un sistema de clasificación serológica ${ }^{35}$ que permitió identificar determinados serogrupos involucrados en los casos de diarrea aguda: los denominados E. coli enteropatógenos clásicos (P.e. ECEP 0111, 055, $0119)$ a los que recientemente se han incorpora. do nuevos serogrupos entre ellos ECEP 0142.

El mecanismo de daño de estos ECEP no está totalmente definido pero hay evidencias que muestran que poseen capacidad para adherirse a ciertos. epitelios sin invadirlos y que producen citotoxinas, si bien no enterotoxinas ${ }^{16-17}$, Los niños recién nacidos resultan muy susceptibles a las infecciones por ECEP. La Unidad de Neonatología del Hospital del Salvador tiene capacidad para 70 pacientes: aproximadamente $50 \%$ son R.N. de bajo peso, algunos requieren de cuidados intensivos y el empleo de procedimientos invasores que representan riesgos per anentes de infecciones intrahospitalarias. 
En los primeros meses de 1982 llamó la atención un aumento desusado de las infecciones intrahospitalarias, la mayoria de ellas consistía en casos de diarrea aguda prolongada con grave compromiso netabólico. Por limitaciones de recursos durantes esos primeros meses, no fue posible efectuar serotinificación de ECEP, por lo cual los casos se trataron empiricamentes con Neomicina, con respuesta insatisfactoria.

Al adquirir el brote carácter francamente epidémico, se logró aislar con la colaboración del I.S.P. el serogrupo 0142 de ECEP en 14 pacientes. Este resultó sensible a Furazolidona y resistente a Neomicina, siendo legítimo suponer que todos los casos eran causados por el mismo agente. Desde entonces cada caso de diarrea se trató con Furazolidona y se aisló, procediendo de igual forma con los contactos (pacientes de la misma sala). Se efectuó un control bacteriológico diario en el Servicio de Distribución de Leche (SEDILE) incluyendo leches maternizadas comerciales y leche materna. Se revisaron las técnicas de atención de enfermería, se reforzó el manejo correcto de los pacientes con enfasis en el lavado de manos, en todo el personal con acceso a la unidad.

No fue posible identificar portadores adultos de ECEP debido a los problemas tècnicos que ello involucra, especialmente en la toma de muestras, pero podemos suponer que un portador transitorio ó permanente entre el personal pudo ser el punto de partida de una infección que se habría difundido entre los recién nacidos a través de las manos del personal, con una elevada tasa de ataque.

Es importante destacar la gravedad que puede alcanzar una infección por ECEP en este periodo de la vida, dadas las relativas deficiencias inmunitarias del huésped y la alta difusibilidad del agente en el ambiente hospitalario. Es interesante tambiên el hecho de haberse aislado E. coli 0142 del hemocultivo de un R.N., ello significa que en estos pacientes, bacterias que tradicionalmente se limitan a provocar patología en el intestino, pueden invadir el torrente circulatorio originando bacteremias o septicemias, situación absolutamente excepcional. Además las alteraciones intestinales provocadas por estos ECEP, en algunos pacientes de algún modo podrían contribuir a la aparición de septicemias por otras enterobacterias como ocurtió en cuatro de nuestros casos.

Por otra parte, si bien la mortalidad fue reducida, el impacto sobre la curva ponderal y la prolongación de los días de hospitalización fueron notorios, produciendo acumulación de pacientes en el servicio y aumentando los riesgos de infecciones secundarias.
Nos parece importante destacar la relación observada entre la gravedad de la infección y el tipo de atimentación; los niños alimentados con leche materna en forma exclusiva o mixta, presentaron significativamente menor incidencia de diarrea y en ninguno de ellos ocurnieron complicaciones como ECN o septicemia. Esta observación reafirmaria el beneficio de la alimentación natural en la prevención de infecciones del recién nacido, tal vez mediante el aporte de elementos importantes en la respuesta inmune pero deficitarios en esta época de la vida, como las IgA secretoras.

Para disminuir el riesgo de morbimottalidad por infecciones intrahospitalarias, entre ellas las diarreas infecciosas en R.N. de bajo peso, siguen teniendo plena vigencia las técnicas rigurosas de asepsia en el manejo de los pacientes, especialmente el lavado de manos, la vigilancia epidemiológica y bacteriológica permanentes y la alimentación con leche materna.

\section{RESUMEN}

Se analizan las características clínicas y epidemiológicas de un brote de diarrea aguda causada por E. coli enteropatógeno clásico (ECEP) serogrupo 0142 en uv servicio de recién nacidos.

En un período de 8 meses se presentaron 70 casos de diarreas agudas, en 22 pacientes se efectuó serotipificación de ECEP y se aisló en 14 de ellos E. coli serogrupo 0142; la pesquisa de Salmonella y Shigella fue negativa en $\operatorname{los} 70$. Del total de casos $92,8 \%$ correspondían a recién nacidos de pretérmino, el $85,7 \%$ de los pacientes desarrollaron un cuadro grave con diarrea prolongada, deshidratación y acentuado compromiso metabólico. La mortalidad fue del $2,9 \%$.

Se discute la interacción entre ECEP y sus factores patogénicos con un huésped con inmu. nodeficiencia relativa como es el recién nacido. La alimentación materna se observó como una elemento protector significativo en el brote descrito.

\section{AGRADECIMIENTOS}

Nuestros sinceros agradecimientos a la Dra. Karin D'Ottone, Jefe del Centro Nacional de Referencia de En terobacterias, del L.S.P., quien seropitificó los ECEP y al Dr. Engenio Spencer del Institu to de Nutrición y Tecrología de los Alimentos (INTA), quien realizó el estudio de rotavirus. 


\section{REFERENCIAS}

1 Hering, E., Fuenzalido, O., Lyneh, B., Prodo, V. Análisis Clínico bacteriológico de un brote de infección por Samonella bredeney en recién nacido, Rev. Chil. Ped. 50: 81, 1979.

2 Cohen, J., Marambio, E., Lynch, B, et al Intoxicación Alimentaria por Bacillus cereus en una Unidad de Reccién Nacidos. Jomadas Pediátricas. Puerto Montt 1982.

${ }^{3}$ Giles, C., Sangster, G., Smith, J. Epidemic gastroenteritis in infants in Aberdeeen during 1947. Arch. Dis. Child. 24: 45, 1949.

${ }^{4}$ Pessoa, G.V.A., Sirguimori, R.T., Irino K. et al. Isolamento de enterobacterias patogénicas en bercarios do Municipio de Sao Paulo. Rev. Inst. Adolfo LeItz 40: $107,1980$.

5 Prado, V. Mimica I. Estudio comparativo doble ciego de los efectos terapéuticos de Neomicina, Lactobacilo versus. Placebo en Infección enteral por Fscherichia coli Enteropatógeno: Rev. Chil. Ped. 45: 143,1974

' Corey G., Algunos aspectos etiológicos y epidemiológicos del Síndrome diarreico agudo irfantil. Rev. Chil. Ped. 46: 1251, 1975.

${ }^{7}$ Errózuriz, O, GlJordino, J., Pino, M. Diarrea aguda del lactante. Fstudio clínico bacteriológico comparativo en grupos tratados con Bactrim, Furoxona y sin medicamento antiinfecciosa oral. Rev. Chil. Ped. 45: $209,1974$.
${ }^{8}$ Figueroa, G. Estudio de prevalencia de serotipos enteropatógenos de $\mathrm{E}$. coli en deposiciones de nirĩos q4느 habitan una poblaciön suburnaba de Santiago. Rev. Chil. Ped 51: 225, 1980.

${ }^{9}$ Zacarias, J., Spencer, E., Prado, V., Herskovic P. er al. Recuperación fecal de rotavirus y otros enteropatógenos en lactantes menores de un año con diarrea aquda. Rev. Chil. Ped. 53:111, 1982.

10 Edwords, P.R., and Ewing W,A,: Identification of Enterobacteriaced. 3rd. Burgess Publishing Co. Min neapolis.

11 Corry, J., Roberts, D. and Skinner, F.: Isolation and identification methods for food poisoning organisms. Academic Press. London 1982.

12 I $C M F$.: Microorganisms in foods 1 . Il $\mathrm{Ed}$ Toronto Press. Toronto 1978.

13 Calderon, A., Macaya, J., Avendaho L.F., et at. Diarrea aguda por rotavirus. Algunos aspectos clínicos. Rev. Chil. Ped. 51: 113, 1980.

14 Bray, $J$, : Isolation of antigenically homogenus strains of Bact. coli neopolitanum from summer diarrhea in infants. J. Path. Belt. $57: 239,1945$.

15 Kauffmonn, F., Orskov, F.: Die bakteriologie der Fscherichia colienteritis. In Säergligo-nenteritis. A Adam, George Thieme Verlag, Stuttgart, 1956.

16 Klipstein, F.A., Rowe, R.F., Engert, H.B., et al.: Enterotoxigenicity of enteropathogenic serotypes of Escherichia coli isolated from infants with cpidemic diarthea. lnfect. Jnmun. 21: 1171, 1978.

17 Konowalchuk, I. Speirs, J.I., Stavrie S.: Vero response to a cy totoxin of Escherichia coli. Infect. Inmun. 18: 775,1977 . 
The Nephrotic Syndrome in children. Prediction of histophatology from clinical and Laboratory charac. teristics at the time of diagnosis Kidney Int. 13: $159,1978$.

10 Hobib R., Gubler M.L.: Les lésions glomerulaires focales des syndromes néphrotiques jdiopathiques de l'enfant. Nephron B: 382, 1971 .

11 Wite R.H.R.; Glosgow E.F.; Mills R.J.: Clinicopathological study of nephrotic Syndrome in chilhood. Lancet IJ $1353,1970$.

12 Sindrome Nefrótico: Normas de Diagnósticos y Manejo. Rev. Chil. Ped. S4: 1, 1983. 